$\underline{\text { Preprint typeset in JHEP style. - HYPER VERSION }}$

SU-ITP-00-30

DAMTP-2000-130

hep-th/0011225

November 10, 2018

\title{
Brane World Sum Rules
}

\author{
Gary Gibbons \\ DAMTP, CMS, Cambridge Univ., Wilberforce Road, Cambridge CB3 0WA, UK \\ E-mail: G.W.Gibbons@damtp.cam.ac.ul \\ Renata Kallosh \\ Department of Physics, Stanford University, Stanford, CA 94305, USA \\ E-mail: kallosh@stanford.edu
}

\begin{abstract}
Andrei Linde
Department of Physics, Stanford University, Stanford, CA 94305, USA

E-mail: iinde@physics.stanford.edu
\end{abstract}

\begin{abstract}
A set of consistency conditions is derived from Einstein equations for brane world scenarios with a spatially periodic internal space. In particular, the sum of the total tension of the flat branes and the non-negative integral of the gradient energy of the bulk scalars must vanish. This constraint allows us to make a simple consistency check of several models. We show that the two-brane Randall-Sundrum model satisfies this constraint, but it does not allow a generalization with smooth branes (domain walls), independently of the issue of supersymmetry. The Goldberger-Wise model of brane stabilization has to include the backreaction on the metric and the fine tuning of the cosmological constant to satisfy the constraints. We check that this is achieved in the DeWolfe-Freedman-Gubser-Karch scenario. Our constraints are automatically satisfied in supersymmetric brane world models.
\end{abstract}

KEYwORDs: eld.pbr.ctg.sgm. 


\section{Contents}

1. Introduction 1

2. Derivation of Sum Rules from Einstein Equations 2

3. Applications of consistency conditions 6

3.1 Randall-Sundrum two-brane scenario 6

3.2 Goldberger-Wise mechanism of brane stabilization 7

3.3 DeWolfe-Freedman-Gubser-Karch stabilization mechanism 9

3.4 Supersymmetric branes in singular spaces 9

\section{Introduction}

There is a strong interest in the possibility that our universe can be represented as a brane in a higher dimensional space [1, 2]. The number of new brane world models is growing fast. Quite often it is difficult to make any judgement concerning the consistency of these models, not only because they are rather complicated, but also because of many 'plausible' assumptions and approximations made by their authors. One of the greatest problems is that one must ensure that the effective cosmological constant on the brane is smaller than $10^{-120}$ in the Planck units.

Therefore it would be quite useful to have a set of simple and sufficiently general rules which would allow one to test new models. In this paper we will derive a set of consistency conditions which can be helpful in this respect. We will consider some combinations of the Einstein equations for warped metrics in the theory of gravity interacting with a collection of scalar fields. The simple case is the one with an internal manifold without boundary, on which the integral of the total derivative vanish, as when the internal space is spatially periodic. This leads to a number of conditions, particularly for the brane tensions and the gradient energy of scalars. Some of these conditions have been derived before, see e.g. [3, 4, 5], but the most important constraint that we are going to use is new. It shows that the sum of the total tension of the branes and the non-negative integral of the gradient energy of scalars is proportional to the four-dimensional curvature of the branes. For flat branes, corresponding to empty space with zero cosmological constant, this sum must vanish. 
This condition allows us to make a simple consistency check of several popular versions of the brane world scenario. We show, in particular, that whereas the original Randall-Sundrum model with two singular branes [2] satisfies this condition, it does not have a consistent generalization with smooth branes (domain walls), independently of the issue of supersymmetry. We also show that the Goldberger-Wise brane stabilization mechanism [6] has to include the backreaction of the scalar field on the metric and a fine tuning of the cosmological constant to satisfy the constraints. ${ }^{1}$ We will explain how fine tuning is achieved in the DeWolfe-Freedman-Gubser-Karch model [7]. We also verify that this consistency condition is automatically satisfied in the supersymmetric brane world scenario proposed by Bergshoeff, Kallosh, and Van Proeyen [8]. The flatness of the branes in this cases follows from supersymmetry of the bulk and brane action.

\section{Derivation of Sum Rules from Einstein Equations}

Here we present the basic equations for warp factors and compactifications. Consider the $D$-dimensional warped product metric of a $p+1$ dimensional spacetime with coordinates $x^{\mu}$ with a $D-p-1$ "internal" space with coordinates $y^{m}$

$$
d s^{2}=g_{m n}(y) d y^{m} d y^{n}+W^{2}(y) g_{\mu \nu}(x) d x^{\mu} d x^{\nu}
$$

Then the Ricci tensor components of the bulk are related to those in spacetime and the internal space by the formulae

$$
\begin{aligned}
& { }^{(D)} R_{\mu \nu}={ }^{(p+1)} R_{\mu \nu}-\frac{g_{\mu \nu}}{p+1} \frac{1}{W^{p-1}} \nabla^{2}\left(W^{p+1}\right) \\
& { }^{(D)} R_{m n}={ }^{(D-p-1)} R_{m n}-\frac{p+1}{W} \nabla_{m} \nabla_{n} W
\end{aligned}
$$

\footnotetext{
${ }^{1}$ For the sake of clarity and to avoid the possibility of confusion, we point out that for the purposes of this paper "fine tuning" refers to the need to constrain one or more parameters appearing in the classical equations of motion, or equivalently in the classical action functional, in order to obtain a solution of the desired type. The larger the number of parameters the larger the degree of fine tuning. If no solution exists for any physically sensible choice of parameters we say that the model is inconsistent. Although one may use perturbation theory to check for fine tuning this is merely because exact solutions are not available. The idea of fine tuning is exact and does not rest on perturbation theory. Finally, although the considerations of this and related papers may throw some light on the full quantum mechanical problem, they operate at best with postulated forms of a classical effective action. The relationship between the parameters appearing in the classical effective action and the parameters appearing in some underlying 'fundamental action,' not least because the models are non-renormalizable, remains obscure. The assumption that fine-tuning of effective parameters requires fine tuning of fundamental parameters may or may not be correct. For example in supersymmetric actions it may follow automatically.
} 
where $\nabla_{m}$ and $\nabla^{2}$ are respectively the covariant derivative and Laplacian with respect to the internal metric $g_{m n}$. The Einstein equations in the bulk are

$$
{ }^{(D)} R_{M N}=8 \pi G_{D}\left(T_{M N}-\frac{1}{D-2} g_{M N} T_{C}^{C}\right),
$$

where $T_{M N}$ is the energy momentum tensor of the matter and $G_{D}$ is the bulk Newton's constant.

If the internal space is "closed," i.e. compact without boundary, then by assuming some general properties of the energy momentum tensor, multiplying the equations by suitable functions of the warp factor $W$ and integrating over the internal space one obtains some necessary conditions for compactification with a flat (or curved) spacetime.

In many cases the singular sources are introduced at $y=y_{i}$ by adding brane actions to the bulk action of the form:

$$
S_{b r}=-\sum_{\alpha} \int d^{p+1} x \sqrt{-\operatorname{det} g_{\mu \nu}} \lambda_{\alpha}(\Phi) .
$$

The curvature as well as the energy-momentum tensor will have some singular contributions of the form $\sim \delta\left(y-y_{i}\right)$. We will consider examples below.

A Special case of $D=5, p=3$ with flat internal metric $g_{y y}=1$ is relevant for domain walls in a 5 -dimensional spacetime. The basic equations reduce to

$$
\begin{gathered}
{ }^{(5)} R_{\mu \nu}={ }^{(4)} R_{\mu \nu}-\frac{\eta_{\mu \nu}}{4} \frac{1}{W^{2}}\left(W^{4}\right)^{\prime \prime} \\
{ }^{(5)} R_{55}=-\frac{4}{W} W^{\prime \prime} .
\end{gathered}
$$

We work with mostly + metric and the ' stands for derivative with respect to $y$.

We may rewrite these equations as follows

$$
\begin{aligned}
{ }^{(5)} R_{\mu}{ }^{\mu}-R_{g} W^{-2} & =-12\left(W^{\prime}\right)^{2} W^{-2}-4 W^{\prime \prime} W^{-1}, \\
{ }^{(5)} R_{5}{ }^{5} & =-4 W^{\prime \prime} W^{-1} .
\end{aligned}
$$

Here we have introduced the notation $R_{g}$ for the curvature scalar of the 4-dimensional space with the metric $g_{\mu \nu}$.

$$
{ }^{(4)} R_{\mu}{ }^{\mu}=R_{g} \text {. }
$$

From eqs. (2.8), (2.9), the bulk curvature ${ }^{(5)} R$ is related to the brane curvature by

$$
{ }^{(5)} R=R_{g} W^{-2}-12\left(W^{\prime}\right)^{2} W^{-2}-8 W^{\prime \prime} W^{-1} .
$$

One may take linear combination of Eq. 2.8) multiplied by $(1-n) W^{n}$ and Eq. (2.9) multiplied by $(n-4) W^{n}$ to obtain:

$$
\left(A^{\prime} e^{n A}\right)^{\prime}=\frac{\left(W^{n}\right)^{\prime \prime}}{n}=\frac{1-n}{12} W^{n}\left({ }^{(5)} R_{\mu}{ }^{\mu}-R_{g} W^{-2}\right)+\frac{n-4}{12} W^{n}{ }^{(5)} R_{5}{ }^{5},
$$


where $W=e^{A}$. Using the Einstein eqs. ${ }^{(5)} R_{A B}=8 \pi G_{5}\left(T_{A B}-\frac{1}{3} g_{A B} T_{C}^{C}\right)$, we can express the desired combination of Ricci tensors in terms of the energy-momentum tensor. We note that $\frac{1}{8 \pi G_{5}} R_{\mu}{ }^{\mu}=-\frac{1}{3} T_{\mu}{ }^{\mu}-\frac{4}{3} T_{5}{ }^{5}$ and $\frac{1}{8 \pi G_{5}} R_{5}{ }^{5}=-\frac{1}{3} T_{\mu}{ }^{\mu}+\frac{2}{3} T_{5}{ }^{5}$. Thus for solutions of the Einstein eqs. with the warped metric (2.1) to be consistent the following local equality must take place

$$
\left(A^{\prime} e^{n A}\right)^{\prime}=\frac{2 \pi G_{5}}{3} e^{n A}\left(T_{\mu}^{\mu}+(2 n-4) T_{5}^{5}\right)-\frac{1-n}{12} W^{n-2} R_{g} .
$$

If the internal space is not closed, this condition may be still useful since it shows that some combination of the energy-momentum tensor is a total derivative when the Einstein equations are satisfied. With proper care of the boundary conditions it provides a consistency condition.

From now on we will concentrate on investigation of compact internal spaces without boundary (as in cases of a spatially periodic metric [2]). In this case the integral of the LHS vanishes. This gives a constraint

$$
\oint e^{n A}\left(T_{\mu}{ }^{\mu}+(2 n-4) T_{5}^{5}\right)=\frac{1-n}{8 \pi G_{5}} R_{g} \oint e^{(n-2) A}
$$

which can be used for all brane worlds with a spatially periodic metric. In case of a flat brane, when $R_{g}=0$ the RHS of the eq. (2.14) vanishes. The most interesting cases are $n=0,1,4$. For $n=0$

$$
\oint\left(T_{\mu}^{\mu}-4 T_{5}^{5}\right)=\frac{1}{8 \pi G_{5}} R_{g} \oint e^{-2 A} .
$$

This equation turns out to be particularly useful for the study of the fine tuning required in the stabilization of the distance between the branes. For $n=1$

$$
\oint e^{A}\left(T_{\mu}^{\mu}-2 T_{5}^{5}\right)=0 .
$$

The right-hand-side of this constraint vanishes in the special case that $n=1$ even for the non-flat brane! The constraint with $n=1$ for flat branes with $R_{g}=0$ has been derived previously ${ }^{2}$ in 4 . However it was suggested there that this constraint is necessary for the vanishing of the four-dimensional cosmological constant. As we see here, this particular constraint at $n=1$ does not differentiate a flat from a non-flat brane. For $n=4$

$$
\oint e^{4 A}\left(T_{\mu}^{\mu}+4 T_{5}^{5}\right)=-\frac{3}{8 \pi G_{5}} R_{g} \oint e^{2 A}=-\frac{3}{8 \pi G_{4}} R_{g} .
$$

This constraint was derived and analysed before in [5]. It was shown there that it is satisfied in the RS model [2].

\footnotetext{
${ }^{2}$ In [9] a different constraint is suggested: the dependence on energy-momentum tensor is as in our eq. (2.16) but the dependence on the metric is different, which seems to contradict 4 as well as our constraints.
} 
It will be useful the give an explicit form of the energy-momentum tensor for the class of theories we will be looking at. The Einstein-Hilbert action is

$$
S_{E G}=\int_{M} d^{5} x \sqrt{-G}\left(2 M^{3} R-\frac{g_{I J}}{2} \partial_{M} \Phi^{I} \partial^{M} \Phi^{J}-V(\Phi)-\sum_{\alpha} \lambda_{\alpha}(\Phi) \delta\left(y-y_{\alpha}\right)\right) 2
$$

where $4 M^{3}=\left(8 \pi G_{5}\right)^{-1}$. In the Randall-Sundrum scenario [2] the mass parameter $M$ is not much different from the $4 \mathrm{~d}$ Planck mass $M_{p}$. The interesting combinations of the energy-momentum tensor are

$$
T_{\mu}^{\mu}=-4\left(\frac{1}{2} \Phi^{\prime} \cdot \Phi^{\prime}+V(\Phi)+\sum_{\alpha} \lambda_{\alpha}(\Phi) \delta\left(y-y_{\alpha}\right)\right),
$$

and

$$
T_{5}^{5}=\frac{1}{2} \Phi^{\prime} \cdot \Phi^{\prime}-V(\Phi) .
$$

Here the indicates a contraction with the curved metric on the scalar moduli space. From equations of motion we find that $2 M^{3} R=-\frac{1}{3} T_{M}^{M}$.

For this class of actions the basic local constraint takes the form

$$
\begin{aligned}
& -\left(A^{\prime} e^{n A}\right)^{\prime}=\frac{1-n}{12} e^{(n-2) A} R_{g} \\
& +\frac{1}{12 \cdot 4 M^{3}} e^{n A}\left((4-n) \Phi^{\prime} \cdot \Phi^{\prime}+2 n V(\Phi)+4 \sum_{\alpha} \lambda_{\alpha}(\Phi) \delta\left(y-y_{\alpha}\right)\right) .
\end{aligned}
$$

For compact internal space without boundary the integral of the left-hand side vanishes and the constraint integrated over the period takes the form

$$
\begin{aligned}
& \oint e^{n A}\left((4-n) \Phi^{\prime} \cdot \Phi^{\prime}+2 n V(\Phi)+4 \sum_{\alpha} \lambda_{\alpha}(\Phi) \delta\left(y-y_{\alpha}\right)\right) \\
& +4 M^{3} \oint(1-n) e^{(n-2) A} R_{g}=0 .
\end{aligned}
$$

There are three particular cases where this constraint may be especially useful.

For $n=1$ one has a condition which is to be satisfied independently of the internal curvature $R_{g}$ of the brane (i.e. independently of the effective four-dimensional cosmological constant):

$$
\oint e^{A}\left(3 \Phi^{\prime} \cdot \Phi^{\prime}+2 V(\Phi)+4 \sum_{\alpha} \lambda_{\alpha}(\Phi) \delta\left(y-y_{\alpha}\right)\right)=0 .
$$

For $n=4$ this gives a constraint that does not include $\Phi^{\prime} \cdot \Phi^{\prime}$ :

$$
\oint e^{4 A}\left(2 V(\Phi)+\sum_{\alpha} \lambda_{\alpha}(\Phi) \delta\left(y-y_{\alpha}\right)-3 M^{3} e^{2 A} R_{g}\right)=0 .
$$

For $n=0$ one finds another constraint, which will be particularly useful for testing the consistency of various models:

$$
\oint\left(\Phi^{\prime} \cdot \Phi^{\prime}+\sum_{\alpha} \lambda_{\alpha}(\Phi) \delta\left(y-y_{\alpha}\right)+M^{3} e^{-2 A} R_{g}\right)=0 .
$$


It is important to realize that this condition does not depend upon the choice of the potential $V(\phi)$, which drops out from this combination. We may rewrite it as follows:

$$
\sum_{\alpha} \lambda_{\alpha}\left(\Phi_{\alpha}\right)+\oint \Phi^{\prime} \cdot \Phi^{\prime}=-M^{3} R_{g} \oint e^{-2 A}
$$

If the geometry on the brane is of de Sitter type, one has $R_{g}>0$ in our notation. In this case one has

$$
\sum_{\alpha} \lambda_{\alpha}\left(\Phi_{\alpha}\right)+\oint \Phi^{\prime} \cdot \Phi^{\prime}<0
$$

If one wants to describe our universe, where $R_{g}$ vanishes with an accuracy of about $10^{-120} M_{P}^{2}$, one can assume, with this accuracy, that $R_{g}=0$. In this case our consistency condition (2.27) becomes

$$
\sum_{\alpha} \lambda_{\alpha}\left(\Phi_{\alpha}\right)+\oint \Phi^{\prime} \cdot \Phi^{\prime}=0
$$

In what follows we will use the last two consistency conditions.

\section{Applications of consistency conditions}

\subsection{Randall-Sundrum two-brane scenario}

In the case of the original Randall-Sundrum scenario with two singular branes and vanishing cosmological constant on the brane, $R_{g}=0$, and without scalar fields [2] this constraint reads

$$
\lambda_{h}=-\lambda_{v}
$$

Here $\lambda_{h}$ and $\lambda_{v}$ correspond to the tension of the hidden and visible branes respectively. The consistency condition is indeed satisfied in [2], the tensions are constant and have opposite signs. This is a fine tuning which is in agreement with our consistency constraint.

If one substitutes the metric with $A=-k|y|$ and $V=\Lambda$ into our constraint (2.23), one finds the second RS condition

$$
\Lambda=k \lambda_{v}
$$

This condition represents the second fine tuning required in the RS scenario.

On the other hand, our constraint (2.28) shows that a smooth generalization of the flat $\left(R_{g}=0\right)$ periodic 2-brane Randall-Sundrum solutions [8] or 3-brane KMPRS solutions 10$]$ without singular sources does not exist. Indeed at $\sum_{\alpha} \lambda_{\alpha}\left(\phi\left(y_{\alpha}\right)\right)=0$ the constraint is reduced to

$$
\oint \Phi^{\prime} \cdot \Phi^{\prime}=0
$$

This is not possible since the LHS is positive and the RHS is strictly zero. The constraint is satisfied only for constant scalars which cannot build a smooth brane. 
For $R_{g}>0$ the corresponding condition becomes $\oint \Phi^{\prime} \cdot \Phi^{\prime}<0$, which is even more difficult to satisfy.

A similar no-go theorem has been established in [11] for the non-singular supersymmetric generalizations of the one-brane Randall-Sundrum scenario [12]. As we see, for the two-brane scenario the no-go theorem can be established independently of the issue of supersymmetry. Thus in what follows we will assume that the branes are singular.

\subsection{Goldberger-Wise mechanism of brane stabilization}

In presence of scalars and singular sources the constraint (2.28) shows that the total tension of the branes must be strictly negative and equal to the volume integral of the gradient energy of the scalars with the opposite sign:

$$
-\sum_{\alpha} \lambda_{\alpha}\left(\Phi\left(y_{\alpha}\right)\right)=\oint \Phi^{\prime} \cdot \Phi^{\prime}>0
$$

We would like to check whether this consistency condition is satisfied in existing models of singular branes on orbifolds stabilized in the presence of scalars.

In the original version of the two-brane Randall-Sundrum scenario, the distance between the branes could take any value. Goldberger and Wise proposed a simple stabilization mechanism for the distance between the branes [6]. They added to the original Randall-Sundrum model with brane tensions $\lambda_{h}=-\lambda_{v}$ a scalar field $\Phi$ with bulk action

$$
S_{b}=\frac{1}{2} \int d^{4} x \int_{-\pi}^{\pi} d y \sqrt{G}\left(G^{A B} \partial_{A} \Phi \partial_{B} \Phi-m^{2} \Phi^{2}\right)
$$

where $G_{A B}$ is the metric in the Randall-Sundrum scenario. They also included interaction terms on the hidden and visible branes (at $y=0$ and $y=\pi$ respectively) given by

$$
S_{h}=-\int d^{4} x \sqrt{-g_{h}} \gamma_{h}\left(\Phi^{2}-v_{h}^{2}\right)^{2}, \quad S_{v}=-\int d^{4} x \sqrt{-g_{v}} \gamma_{v}\left(\Phi^{2}-v_{v}^{2}\right)^{2}
$$

where $g_{h}$ and $g_{v}$ are the determinants of the induced metric on the hidden and visible branes respectively, and $\gamma_{h}$ and $\gamma_{v}$ are some positive coupling constants. An investigation of this system for large positive constants $\gamma_{v}$ and $\gamma_{h}$ has shown that $\Phi(0) \approx v_{h}, \Phi(\pi) \approx v_{v}$, and the distance $r$ between the branes becomes stabilized at

$$
r_{c} \approx\left(\frac{4}{\pi}\right) \frac{k}{m^{2}} \ln \left[\frac{v_{h}}{v_{v}}\right]
$$

A certain advantage of this mechanism is that the brane stabilization occurs even if one changes a bit the bulk cosmological constant in the RS scenario. Thus at least one of the two fine tunings of the RS model can be relaxed.

However, a detailed investigation of the brane stabilization was performed in [6] without taking into account the back reaction of the scalar field on the metric. 
Our consistency conditions in the form (2.27), (2.28) allow us immediately to check whether this approximation is consistent.

To use eq. (2.28) in any model of the brane world with branes located at the fixed points of an $S^{1} / \mathbf{Z}_{2}$ orbifold all we have to know is that the total negative tension of the branes compensates exactly the positive integral of the gradient terms of scalars. In the Goldberger-Wise model our constraint (2.28) takes the form

$$
\int_{-\pi}^{\pi} d y \Phi^{\prime} \cdot \Phi^{\prime}+\left[\lambda_{h}+\lambda_{v}+\gamma_{h}\left(\Phi^{2}(0)-v_{h}^{2}\right)^{2}+\gamma_{v}\left(\Phi^{2}(\pi)-v_{v}^{2}\right)^{2}\right]=0 .
$$

In the original version of the RS model without stabilization [2] one needs to impose the fine tuning condition $\lambda_{h}+\lambda_{v}=0$. Thus our constraint (2.28) was satisfied. The Goldberger-Wise mechanism (in the simplest approximation when the backreaction is neglected) presumes that the condition $\lambda_{h}+\lambda_{v}=0$ still holds. But then the constraint cannot be satisfied because there are three new positive contributions which do not cancel: the term $\int_{-\pi}^{\pi} d y \Phi^{\prime} \cdot \Phi^{\prime}$ and the terms $\gamma_{h}\left(\Phi^{2}(0)-v_{h}^{2}\right)^{2}$ and $\gamma_{v}\left(\Phi^{2}(\pi)-v_{v}^{2}\right)^{2}$.

The term $\int_{-\pi}^{\pi} d y \Phi^{\prime} \cdot \Phi^{\prime}$ is strictly positive because the field $\Phi$ changes from (approximately) $v_{h}$ to $v_{v}$, and the distance $r_{c}$ is nonzero only if $v_{h} \neq v_{v}$. Thus $\Phi^{\prime} \neq 0$, and $\int_{-\pi}^{\pi} d y \Phi^{\prime} \cdot \Phi^{\prime}>0$. One could expect that the terms $\gamma_{h}\left(\Phi^{2}(0)-v_{h}^{2}\right)^{2}$ and $\gamma_{v}\left(\Phi^{2}(\pi)-v_{v}^{2}\right)^{2}$ may vanish. However, the field $\Phi$ is not exactly equal to $v_{h}$ and $v_{v}$ on the branes. One can show that in the large coupling constant approximation used in [6] one has $\gamma_{h}\left(\Phi^{2}(0)-v_{h}^{2}\right)^{2}=\frac{m^{4}}{64 k^{2} \gamma_{h}}$, and $\gamma_{v}\left(\Phi(\pi)^{2}-v_{v}^{2}\right)^{2}=\frac{m^{4}}{64 k^{2} \gamma_{v}}$. These terms vanish in the limit $\gamma_{h}, \gamma_{v} \rightarrow \infty$, in agreement with [6]. However, for any finite $\gamma_{h}$ and $\gamma_{v}$ these terms are positive. Thus all three terms discussed above are strictly positive, which violates the consistency condition (3.8).

Goldberger and Wise discussed the possibility to alter the Randall-Sundrum condition $\lambda_{h}+\lambda_{v}=0$ (3.8) in order to find a self-consistent solution. Our constraint implies that to achieve consistency one should take $\lambda_{h}+\lambda_{v}<0$. One may argue that a small change of brane tensions should not destabilize the system, but it should affect $R_{g}$. Then by making $\lambda_{h}+\lambda_{v}$ slightly negative one will still have a stable two-brane system. By gradually decreasing $\lambda_{h}+\lambda_{v}$ one may eventually achieve the desirable regime $R_{g}=0$ for some value of $\lambda_{h}+\lambda_{v}$.

This would imply a more elaborate fine tuning of $\lambda_{h}+\lambda_{v}$ than in the RS model, where one simply required $\lambda_{h}=-\lambda_{v}$. Here one would need to find a solution of a combined system of equations for the metric and for the scalar field, and only after that one would know whether a consistent solution with flat branes exists and what kind of fine tuning is actually required. To check the consistency of the solution one would need to verify not only the simplest constraint (2.26), but also (2.23), as we did for the RS model. 


\subsection{DeWolfe-Freedman-Gubser-Karch stabilization mechanism}

A consistent realization of the Goldberger-Wise scenario for a certain class of potentials of the bulk scalars was proposed by DeWolfe, Freedman, Gubser, and Karch [7]. In their model the first brane is placed at $r=0$, the second brane at $r=r_{0}$, and the point $2 r_{0}$ is identified with the point $r=0$ so that $\int_{0}^{2 r_{0}} d r \Phi^{\prime} \cdot \Phi^{\prime}=2 \int_{0}^{r_{0}} d r \Phi^{\prime} \cdot \Phi^{\prime}$. The constraint (2.26) takes the form

$$
\lambda_{1}\left(\Phi_{1}\right)+\lambda_{2}\left(\Phi_{2}\right)=-2 \int_{0}^{r_{0}} d r\left(\Phi^{\prime} \cdot \Phi^{\prime}+M^{3} e^{-2 A} R_{g}\right) .
$$

The solution for the flat branes with $R_{g}=0$ has the following property: $\Phi^{\prime}=\frac{1}{2} \frac{\partial W}{\partial \Phi}$ in the volume between the first and the second brane. The scalar dependent tensions of the brane are chosen as follows ${ }^{3}$ :

$$
\lambda_{1}\left(\Phi_{1}\right)=W\left(\Phi_{1}\right), \quad \lambda_{2}\left(\Phi_{2}\right)=-W\left(\Phi_{2}\right)
$$

The potential is presented via a 'superpotential' $W$ as $V(\Phi)=\frac{1}{8}\left(\frac{\partial W}{\partial \Phi}\right)^{2}-\frac{1}{3} W(\Phi)^{2}$. The choice of the 'superpotential' $W(\Phi)=\frac{3}{L}-b \Phi^{2}$ is not given by any known supersymmetric theory. However, this choice is useful for providing an explicit example of the flat brane solution with the vanishing cosmological constant ${ }^{4}$. Performing the integral in eq. (3.9) one finds that the constraint requires that

$$
b\left(\Phi_{1}^{2}-\Phi_{2}^{2}\right)+\lambda_{1}\left(\Phi_{1}\right)+\lambda_{2}\left(\Phi_{2}\right)=0 .
$$

Here $b r_{0}=\ln \left(\Phi_{1} / \Phi_{2}\right)$. The distance between the branes $r_{0}$ is positive and therefore for $b>0, \Phi_{1}>\Phi_{2}$ and for $b<0, \Phi_{2}>\Phi_{1}$. This explains the fine tuning condition $\lambda_{1}\left(\Phi_{1}\right)+\lambda_{2}\left(\Phi_{2}\right)=W\left(\Phi_{1}\right)-W\left(\Phi_{2}\right)$ required in [7] for the vanishing of the cosmological constant. Indeed the positive contribution from the volume integral $b\left(\Phi_{1}^{2}-\Phi_{2}^{2}\right)>0$ is precisely cancelled by the negative total tension of the branes $\lambda_{1}\left(\Phi_{1}\right)+\lambda_{2}\left(\Phi_{2}\right)=$ $b\left(\Phi_{2}^{2}-\Phi_{2}^{1}\right)$. We checked that other consistency conditions are also satisfied in this model.

Since the theory proposed in [7] is not supersymmetric, one should take special care to ensure vanishing (or smallness) of the cosmological constant with an account taken of radiative corrections.

\subsection{Supersymmetric branes in singular spaces}

As we see, simple consistency checks can be very helpful and can bring unexpected results when analyzing popular versions of the brane world scenario. So far, we have applied these checks to the simplest five-dimensional non-supersymmetric models.

\footnotetext{
${ }^{3}$ The relation between the brane tension and the superpotential which allows the jump conditions to be satisfied was first suggested in 13 in the context of $N=8$ gauged supergravity.

${ }^{4}$ The issue of the stabilization of the distance between the branes is more subtle than in [6] as it requires an additional assumption on the relation between $\lambda_{i}^{\prime \prime}\left(\Phi_{i}\right)$ and $W^{\prime \prime}\left(\Phi_{i}\right)$.
} 
For a while it was not clear whether a supersymmetric brane world theory is possible at all. Until very recently, a complete supersymmetry had not been formulated in singular spaces, so all attempts have been focused on the possibility of obtaining branes as non-singular domain wall solutions of supergravity theories. However, after a series of no-go theorems [11], the attention shifted towards the possibility of formulating five-dimensional supergravity in singular spaces. The first example of such supersymmetric theory involving a scalar and a vector fields was proposed in [8] ${ }^{5}$. Consistency of this theory was achieved without fine tuning, but in a very nontrivial way. It was necessary to extend the gauged five-dimensional supergravity to include the five-form field strength $F=d A+\ldots$ which on shell is piece-wise constant. The gauge coupling constant $g$ was promoted to a field $G(x)$. When the equations of motion from the bulk and the brane actions are solved for the potential $A$, the gauge coupling field becomes, on shell, piece-wise constant, changing the sign across the wall: $G(x)=g \epsilon(x)$. Branes are sources of five-form fluxes which are absent in the standard formulations of the five-dimensional gauged supergravities.

The supersymmetric brane action depends on the induced metric $g_{(4)}$, on the superpotential of the scalar fields $W$ and on the projection of the potential $A_{\mu \nu \rho \sigma}$ in the brane directions:

$$
\mathcal{L}_{\text {brane }}=-2 g\left(\delta\left(x^{5}\right)-\delta\left(x^{5}-\tilde{x}^{5}\right)\right)\left(\sqrt{g}_{(4)} 3 W(\phi)+\frac{1}{4 !} \varepsilon^{\mu \nu \rho \sigma} A_{\mu \nu \rho \sigma}\right) .
$$

This bosonic action is supersymmetric because the variation of the two terms combines into a fermionic field which vanishes on the brane as the result of $\mathbf{Z}_{2}$ symmetry. Thus the form of the brane action and of the brane tensions are determined by supersymmetry.

Here we will make an additional consistency check of this theory using our methods. It was shown in [8] that the bulk and brane supersymmetry with the orbifold condition requires that the tensions of the branes are related to the superpotential as follows: $\lambda_{1}\left(\Phi_{1}\right)=3 g W\left(\Phi_{1}\right), \lambda_{2}\left(\Phi_{2}\right)=-3 g W\left(\Phi_{2}\right)$. This condition, up to a normalization, is exactly the fine tuning condition (3.10) of Ref. [7]. The main difference is that in [8] this condition appears automatically as a result of supersymmetry in singular spaces.

We can calculate the volume integral of the gradient energy using the BPS relation between the derivative of the scalars and the derivative of the superpotential with respect to the scalars, which leads to

$$
\Phi^{\prime} \cdot \Phi^{\prime}=3 G(x) W^{\prime} .
$$

Thus the integral of the gradient energy of scalars is given precisely by $3 W\left(\Phi_{2}\right)-$ $3 W\left(\Phi_{1}\right)$ which cancels the expression for the tensions in the constraint equation

\footnotetext{
${ }^{5}$ The domain wall solution of Horava-Witten theory [14] reduced to 5 dimensions in 15] satisfies the consistency condition (2.26). The complete supersymmetry of the embedding theory is, however, not fully understood.
} 
(2.26). Because of the BPS property of the solution which has $1 / 2$ of the unbroken supersymmetry and is derived in the framework of the supersymmetric bulk and brane action, the brane cosmological constant in (2.26) has to vanish. Thus, not surprisingly, supersymmetry in the present context requires a flat brane solution without any need for fine tuning.

In conclusion, we have derived some simple consistency conditions for brane solutions in spatially periodic internal space for which the integrals of total derivatives vanish. The simplest one is given in eq. $(\overline{2.26})$. It relates the tensions on two branes to the volume integral of the gradient energy of scalars and the cosmological constant on the brane.

The authors are grateful to D. Freedman and G. Horowitz for valuable discussions and encouragement and to S. Gubser, W. D. Goldberger and M. B. Wise for very useful correspondence and important comments. This work was supported in part by NSF grant PHY-9870115. 


\section{References}

[1] N. Arkani-Hamed, S. Dimopoulos and G. Dvali, "The hierarchy problem and new dimensions at a millimeter," Phys. Lett. B429, 263 (1998), hep-ph/9807344; I. Antoniadis, N. Arkani-Hamed, S. Dimopoulos and G. Dvali, "New dimensions at a millimeter to a Fermi and superstrings at a TeV," Phys. Lett. B436, 257 (1998), hep$\mathrm{ph} / 9804398$.

[2] L. Randall and R. Sundrum, "A large mass hierarchy from a small extra dimension," Phys. Rev. Lett. 83, 3370 (1999) hep-ph/9905221.

[3] G. W. Gibbons, "Aspects of Supergravity Theories," in Supersymmetry, Supergravity and Related Topics, eds. F. del Aguila, J. A. de Azcaragga and L. E. Ibanez, World Scientific (1985).

[4] U. Ellwanger, "Constraints on a brane-world from the vanishing of the cosmological constant," Phys. Lett. B473, 233 (2000) hep-th/9909103.

[5] S. Forste, Z. Lalak, S. Lavignac and H. P. Nilles, "The cosmological constant problem from a brane-world perspective," JHEP 0009, 034 (2000) hep-th/0006139.

[6] W. D. Goldberger and M. B. Wise, "Modulus stabilization with bulk fields," Phys. Rev. Lett. 83, 4922 (1999) hep-ph/9907447.

[7] O. DeWolfe, D. Z. Freedman, S. S. Gubser and A. Karch, "Modeling the fifth dimension with scalars and gravity," Phys. Rev. D62, 046008 (2000) hep-th/9909134.

[8] E. Bergshoeff, R. Kallosh and A. Van Proeyen, "Supersymmetry in singular spaces," JHEP 0010, 033 (2000) hep-th/0007044.

[9] P. Kanti, I. I. Kogan, K. A. Olive and M. Pospelov, "Single-brane cosmological solutions with a stable compact extra dimension," Phys. Rev. D61, 106004 (2000) hep-ph/9912266; I I. I. Kogan and G. G. Ross, "Brane universe and multigravity: Modification of gravity at large and small distances," Phys. Lett. B485, 255 (2000) hep-th/0003074.

[10] I. I. Kogan, S. Mouslopoulos, A. Papazoglou, G. G. Ross and J. Santiago, "A three three-brane universe: New phenomenology for the new millennium?," Nucl. Phys. B584, 313 (2000) [hep-ph/9912552.

[11] R. Kallosh and A. Linde, "Supersymmetry and the brane world," JHEP 0002, 005 (2000) hep-th/0001071; K. Behrndt and M. Cvetic, "Anti-de Sitter vacua of gauged supergravities with 8 supercharges," Phys. Rev. D61, 101901 (2000) hep-th/0001159]; G. W. Gibbons and N. D. Lambert, "Domain walls and solitons in odd dimensions," Phys. Lett. B488, 90 (2000) hep-th/0003197; J. Maldacena and C. Nunez, "Supergravity description of field theories on curved manifolds and a no go theorem," hep-th/0007018. 
[12] L. Randall and R. Sundrum, "An alternative to compactification," Phys. Rev. Lett. 83, 4690 (1999) hep-th/9906064.

[13] A. Brandhuber and K. Sfetsos, JHEP 9910, 013 (1999) hep-th/9908116.

[14] P. Hor̆ava and E. Witten, "Eleven-dimensional supergravity on a manifold with boundary," Nucl. Phys. B475 (1996) 94 hep-th/9603142.

[15] A. Lukas, B. A. Ovrut, K. S. Stelle and D. Waldram, "Heterotic M-theory in five dimensions," Nucl. Phys. B552, 246 (1999) hep-th/9806051. 